
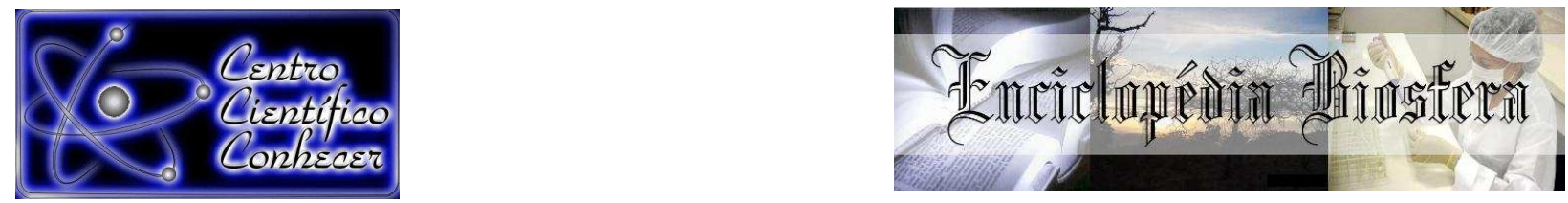

\title{
MORFOMETRIA CORPORAL E CAPACIDADE VOLUMÉTRICA DO ESTÔMAGO DE NEONATOS CANINOS DE PEQUENO, MÉDIO E GRANDE PORTE
}

Andrezza Braga Soares da Silva ${ }^{1^{*}}$, Maria Michele Araújo de Sousa Cavalcante ${ }^{1}$, Ingrid Macêdo de Oliveira ${ }^{2}$, Luana de Oliveira Lopes ${ }^{3}$, Airton Mendes Conde Júnior ${ }^{4}$

1 Mestrandas no Programa de Pós graduação em Ciência e Saúde - UFPI 2 Mestranda no Programa de Pós graduação em Odontologia - UFPI

3 Doutoranda em Biotecnologia (Renorbio) - UFPI

4 Médico Veterinário e Professor Adjunto do Departamento de Morfologia -UFPI *andrezzab1@hotmail.com

Recebido em: 08/09/2015 - Aprovado em: 14/11/2015 - Publicado em: 01/12/2015 DOI: http://dx.doi.org/10.18677/Enciclopedia_Biosfera_2015_023

Objetivou-se estudar a morfometria corporal e a capacidade volumétrica estomacal de caninos neonatos de diferentes portes. Foram avaliados doze neonatos divididos em: Grupol (pequeno porte); Grupoll (médio porte); Grupolll (grande porte), que vieram a óbito pós-parto. Estes foram pesados, medidos e posteriormente dissecados. Procedeu-se com extração e medição do volume estomacal através do preenchimento com leite. Encontrou-se a média e desvio padrão de cada grupo para cada parâmetro. Grupo I: peso 155,15 $\pm 23,53 \mathrm{~g}$; comprimento total $202,33 \pm 24,11 \mathrm{~mm}$; comprimento cranial $59,35 \pm 11,53 \mathrm{~mm}$; comprimento caudal $49,97 \pm 10,82 \mathrm{~mm}$; altura

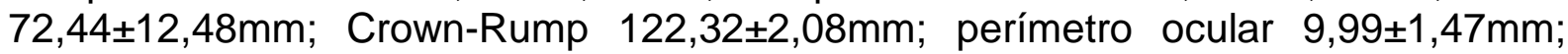
comprimento auricular $15,37 \pm 3,25 \mathrm{~mm}$; comprimento abdominal $53,52 \pm 7,11 \mathrm{~mm}$; diâmetro biparietal 37,16 $\pm 17,98 \mathrm{~mm}$; comprimento torácico 42,33 $\pm 7,31 \mathrm{~mm}$;

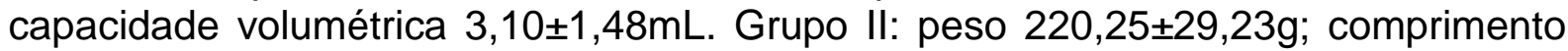
total $194 \pm 62,93 \mathrm{~mm}$; comprimento cranial $53,47 \pm 9,82 \mathrm{~mm}$; comprimento caudal $59,92 \pm 16,75 \mathrm{~mm}$; altura $65,42 \pm 5,15 \mathrm{~mm}$; Crown-Rump 141,72 $\pm 10,87 \mathrm{~mm}$; perímetro ocular 10,68 $\pm 1,67 \mathrm{~mm}$; comprimento auricular $16,07 \pm 2,19 \mathrm{~mm}$; comprimento abdominal $65,59 \pm 10,14 \mathrm{~mm}$; diâmetro biparietal $32,47 \pm 2,51 \mathrm{~mm}$; comprimento torácico $52,88 \pm 2,74 \mathrm{~mm}$; capacidade volumétrica $7,79 \pm 2,61 \mathrm{~mL}$. Grupo III: peso $414,52 \pm 16,09 \mathrm{~g}$; comprimento total $286,5 \pm 5,07 \mathrm{~mm}$; comprimento cranial $83,52 \pm 14,53 \mathrm{~mm}$; comprimento caudal $71,92 \pm 19,87 \mathrm{~mm}$; altura $89,85 \pm 3,39 \mathrm{~mm}$;

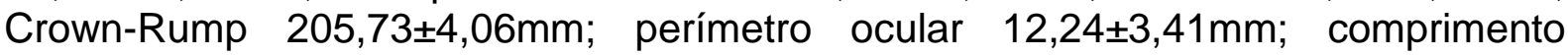
auricular 21,31 $\pm 1,58 \mathrm{~mm}$; comprimento abdominal $65,04 \pm 5,89 \mathrm{~mm}$; diâmetro biparietal $33,77 \pm 9,48 \mathrm{~mm}$; comprimento torácico $53,36 \pm 11,82 \mathrm{~mm}$; capacidade volumétrica $19,70 \pm 3,78$. É possível classificar-los em três portes de acordo com os dados morfométricos. O estômago dos neonatos varia a capacidade volumétrica dependendo do porte do animal.

PALAVRAS-CHAVE: capacidade volumétrica, estômago, morfometria, neonatos caninos. 


\title{
BODY MOPHOMETRY AND STOMACH VOLUMETRIC CAPACITY OF CANINE NEONATES OF SHORT, MEDIUM AND LARGE BREEDS
}

\begin{abstract}
This study aimed to evaluate the volumetric capacity stomach and morphometry of neonates canines. Twelve neonates were obtained and classified in three Groups: Group I (breed short); Group II (breed medium); Group III (breed large), that eventually died postpartum. The animals were classified, wheighed, measured e then dissected. Proceeded with the extraction and measurement of stomach volume by filling with a milk. We found the mean and standard deviation of each group for each parameter. Group I: weight $155.15 \pm 23.53 \mathrm{~g}$, total length $202.33 \pm 24.11 \mathrm{~mm}$ cranial length $59.35 \pm 11.53 \mathrm{~mm}$ caudal length $49.97 \pm 10,82 \mathrm{~mm}$, height $72.44 \pm 12.48 \mathrm{~mm}$; Crown Rump $122.32 \pm 2.08 \mathrm{~mm}$; ocular perimeter $9.99 \pm 1.47 \mathrm{~mm}$, length headset $15.37 \pm 3$, 25mm; abdominal length $53.52 \pm 7.11 \mathrm{~mm}$; biparietal diameter $37.16 \pm 17.98 \mathrm{~mm}$; thoracic length $42.33 \pm 7.31 \mathrm{~mm}$; volumetric capacity $3.10 \pm 1.48 \mathrm{~mL}$. Group II: weight $220.25 \pm 29.23 \mathrm{~g}$, total length $194 \pm 62.93 \mathrm{~mm}$ cranial length $53.47 \pm 9.82 \mathrm{~mm}$; caudal length $59.92 \pm 16.75 \mathrm{~mm}$; height $65.42 \pm 5.15 \mathrm{~mm}$; Crown Rump $141.72 \pm 10.87 \mathrm{~mm}$; ocular perimeter $10.68 \pm 1.67 \mathrm{~mm}$; ear length $16.07 \pm 2.19 \mathrm{~mm}$; abdominal length $65.59 \pm 10.14 \mathrm{~mm}$; biparietal diameter $32.47 \pm 2.51 \mathrm{~mm}$; thoracic length $52.88 \pm 2.74 \mathrm{~mm}$; volumetric capacity $7.79 \pm 2.61 \mathrm{~mL}$. Group III: weight $414.52 \pm 16.09 \mathrm{~g}$; total length $286.5 \pm 5.07 \mathrm{~mm}$; cranial length $83.52 \pm 14.53 \mathrm{~mm}$; caudal length 71.92 $\pm 19.87 \mathrm{~mm}$; height $89.85 \pm 3.39 \mathrm{~mm}$; Crown Rump 205.73 $\pm 4.06 \mathrm{~mm}$; ocular perimeter $12.24 \pm 3.41 \mathrm{~mm}$; ear length $21.31 \pm 1.58 \mathrm{~mm}$; abdominal length $65.04 \pm 5.89 \mathrm{~mm}$; biparietal diameter $33.77 \pm 9.48 \mathrm{~mm}$; thoracic length $53.36 \pm 11.82 \mathrm{~mm}$; volumetric capacity $19.70 \pm 3.78 \mathrm{~mL}$. These data serve as parameters to normalities. The stomach of neonates varies your volumetric capacity according to the animal size in a relation proportional directly.
\end{abstract}

KEYWORDS: canine neonates; morphometry; stomach; volumetric capacity.

\section{INTRODUÇÃO}

O sistema digestório tem a função de transformar macronutrientes em micronutrientes capazes de circular na corrente sanguínea, possibilitando os mecanismos citofisiológicos normais no individuo (AIRES, 2008; GUYTON \& HALL, 2002).

Dentre os órgãos deste sistema, o estômago é o ponto chave para o início da digestão em cães e gatos, onde exerce funções motoras e secretoras durante o processo. Nos caninos, existem pontos críticos durante a vida, onde o sistema digestório é responsável diretamente pela manutenção da glicemia, visto que não existem reservas no organismo suficiente. Um desses períodos compreende até a segunda semana de vida, o qual chamamos de período neonatal (DAVIDSON, 2003; OLIVEIRA et al., 2008). E, a ciência que o estuda denomina-se neonatologia. Esta ciência é de suma importância, por estudar problemas que variam de cuidados pósparto até o estudo de patologias específicas de neonatos. Constitui-se, então, uma especialização em Medicina Veterinária (PASSOS, 2006).

Sabemos, pois, que o neonato, apresentando-se morfofisiologicamente ainda imaturo, necessita de cuidados especiais, por possuir evolução neurológica, imunológica e comportamental dependente deste período (BARRETO, 2003; CRESPILLO et al., 2007). Estudos mostram que de $17 \%$ a $30 \%$ dos recém nascidos morrem com até 15 dias do nascimento. Vários fatores estão envolvidos neste 
percentual, dentre eles citam-se: prolongado trabalho de parto, negligência maternal, falta de cuidados, falta ou excesso de leite, anormalidades congênitas ou adquiridas pós-parto (INDEBRO et al., 2007).

Estudos sobre morfologia e morfometria dos cães neonatos são importantes, pois através destes dados, é possível estabelecer relações que permitem a identificação da origem evolutiva deste grupo, padronizar raças e o desenvolvimento intra-uterino normal, além de elucidar processos fisiopatológicos (KERSWELL et al., 2009). É possível, também, identificar doenças relacionadas à morfologia dos cães, como anormalidades funcionais congênitas ou não (BURBIDGE, 1995; BERCHT, 2009.).

Em alguns casos, onde impossibilidade materna de manter e cuidar da cria são observados, são necessários aconselhamentos de um médico veterinário. Estes incluem cuidados com a manutenção da temperatura corpórea, estímulos de defecação e micção além, é claro, da manutenção das taxas glicêmicas e calóricas por meio da administração de alimento específico para neonatos. (DAVIDSON, 2003).

A alimentação do neonato pode ser feita artificialmente e para isto existem formulações prontas solúveis em água disponíveis para compra. Neste momento, o acompanhamento do médico veterinário para administração da dose correta é de vital importância diante dos problemas que ocorrem pelo excesso ou escassez de alimentos administrados. Esses problemas geram doenças que vão desde hipoglicemia, problemas cardiovasculares, diabetes até pneumonia aspirativa. (TORTOLA, 2008, FURNISS, 2010; CARVALHO et al., 2006).

Estudos morfológicos básicos como avaliação da capacidade volumétrica do estômago de neonatos são necessários para subsidiar o manejo correto do volume de alimento que deve ser administrado ao neonato canino e, dessa forma, evitar a hiper ou a hipo alimentação.

Devido à inexistência de informações sobre o tema abordado, pretende-se, com este estudo, fornecer dados quantitativos relacionados à morfometria externa corporal, volumetria da capacidade gástrica e, ainda, estabelecer a sintopia do estômago na cavidade abdominal de neonatos caninos de portes diferentes, com o intuito de dar ferramentas que permitam aos médicos veterinários neatologistas uma melhor abordagem nutricional para estes filhotes.

\section{MATERIAL E MÉTODOS}

O estudo foi realizado na Universidade Federal do Piauí (UFPI), no Setor de Histologia e Embriologia do Centro de Ciências da Saúde. Através de prévio contato com o Hospital Veterinário da Universidade Federal do Piauí e com clinicas veterinárias particulares obtiveram-se cães neonatos de pequeno, médio e grande porte sem raça definida.

Foram estudados doze neonatos obtidos de fêmeas com partos distócicos ou fêmeas gestantes que tiveram problemas durante o parto e/ou cesariana e, por ventura, os filhotes tenham vindo a óbito. Os animais foram levados ao Laboratório de Histologia e Embriologia da UFPI. Os neonatos foram identificados quanto a sexo, pesados em balança digital de precisão (Marte/AY220) e classificados em três grupos: Grupo I: cães de pequeno porte (100 a 400g); Grupo II: cães de médio porte (peso variando de 200 a 300); Grupo III: cães de grande porte (peso variando de 400 a 500g). (BARRETO 2003). 
Após classificação, os neonatos foram submetidos ao estudo macromorfométrico com auxílio de um paquímetro de precisão (King.Tools502.150BL) e conforme orientações de Mandarim de Lacerda (1994): peso(g), comprimento total $(\mathrm{mm})$, comprimento cranial $(\mathrm{mm})$; comprimento caudal $(\mathrm{mm})$; altura; Crown-Rump (mm); perímetro ocular $(\mathrm{mm})$; comprimento auricular(mm); comprimento abdominal $(\mathrm{mm})$; diâmetro biparietal $(\mathrm{mm})$; comprimento torácico $(\mathrm{mm})$.

Após a obtenção dos dados morfométricos, procedeu-se com a dissecção. Inicialmente foi realizada incisão, com uso de lâmina de bisturi número 24, na cavidade abdominal, através na linha alba para exposição das vísceras abdominais. Após o acesso, identificou-se o omento maior, o qual foi removido cuidadosamente e a disposição das vísceras abdominais foi observada e fotografada com máquina digital fotográfica (SONY/DSC_H50) Posteriormente, o estômago foi localizado e a sintopia estomacal foi estudada relacionada à cavidade na qual esta localizada, assim como às vísceras abdominais. O estômago, então, foi extraído da cavidade, após a realização da ligadura com fio de algodão e da incisão na região cárdica e na pilórica. Após remoção do órgão da cavidade este foi observado quando a morfometria e submetido aos estudos relacionados a sua capacidade volumétrica.

A avaliação da capacidade volumétrica do estômago foi feita pela repleção do órgão após ligadura. Para tanto, utilizou-se uma seringa com capacidade de $10 \mathrm{~mL}$, agulha $(25 \times 0,7)$ repleta de leite (Itambé). O uso do leite foi proposto para mimetizar da melhor forma o processo de administração do produto feito ao neonato. Certificou-se da qualidade da ligadura na válvula cárdia. Assim, o estômago foi preenchido por meio do esfíncter cárdia com o leite até sua total repleção, medida pela turgidez do órgão (Figura 1). Foi confeccionada uma tabela com as capacidades volumétricas dos neonatos. As amostras foram submetidas às análises estatísticas para obtenção de média, desvio padrão e gráficos através do programa Graphpad Prism 3.0.
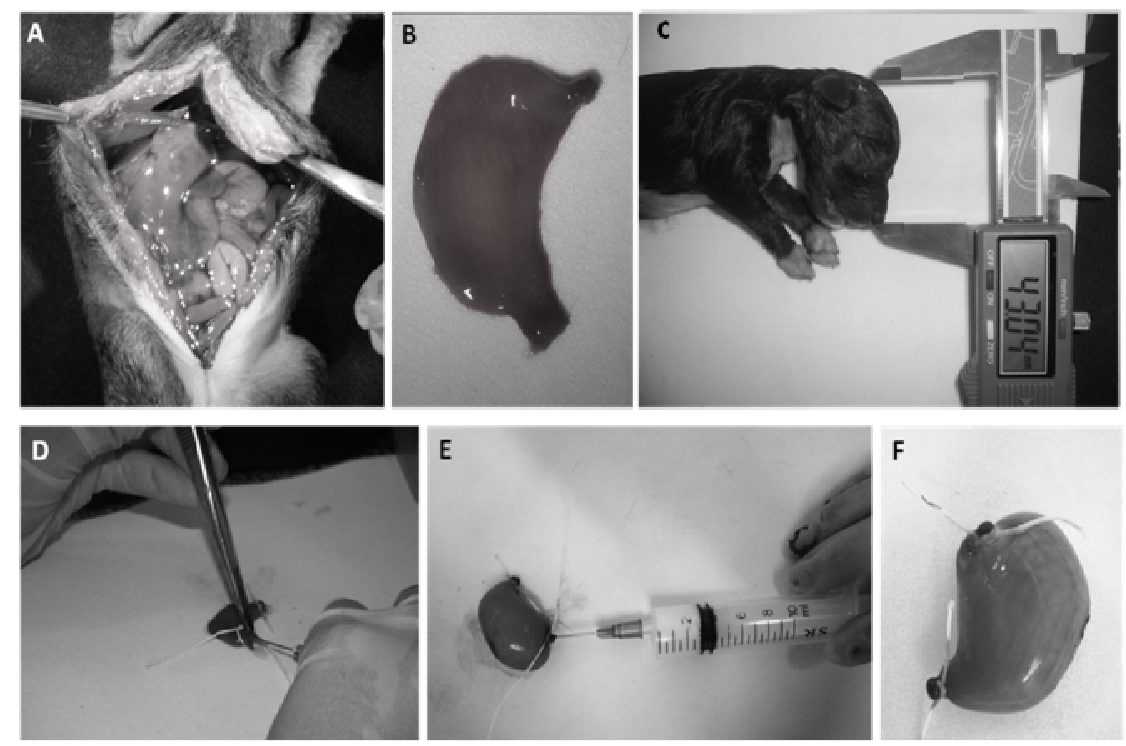

FIGURA 1: Prancha demonstrando a metodologia de obtenção e repleção do estômago de neonatos caninos. Observa-se a identificação, coleta e repleção do estômago. A- sintopia do estômago na cavidade abdominal; B- estômago obtido após dissecção; C- morfometria do órgão com auxilio de paquimetro; D - Acoplamento da agulha pela válvula cárdia para injeção de solução de leite; $\mathrm{E}$ - repleção do órgão para medição da a capacidade volumétrica $\mathrm{F}$ - órgão repleto, em sua capacidade máxima, com leite. Fonte: Arquivo Pessoal 


\section{RESULTADOS E DISCUSSÃO}

No estudo realizado, foi possível, conforme sugerido na literatura (BARRETO, 2003), a divisão dos neonatos caninos em três grupos (animais de pequeno, médio e grande porte) de acordo com o peso. Aqueles de pequeno porte (entre 100 e $200 \mathrm{~g}$ ), médio porte (entre 200 e $300 \mathrm{~g}$ ) e grande porte (entre 300 e $500 \mathrm{~g}$ ).

Os dados morfométricos estão descritos na Tabela 1 segundo a média e desvio padrão encontrados para os neonatos caninos estudados.

TABELA 1: Dados das médias da morfometria corporal e capacidade volumétrica estomacal de neonatos caninos em pequeno, médio e grande porte. Teresina, 2010.

\begin{tabular}{llrr}
\hline & Grupo I & \multicolumn{1}{c}{ Grupo II } & \multicolumn{1}{c}{ Grupo III } \\
\hline Peso(g) & $175,30 \pm 24,76$ & $232,80 \pm 26,98$ & $443,14 \pm 63,04$ \\
Comprimento total(mm) & $190,91 \pm 49,43$ & $225,33 \pm 35,62$ & $294,60 \pm 21,74$ \\
Comprimento cranial $(\mathrm{mm})$ & $49,39 \pm 9,83$ & $52,79 \pm 5,17$ & $68,84 \pm 12,0$ \\
Comprimento caudal $(\mathrm{mm})$ & $53,73 \pm 16,69$ & $70,042 \pm 10,82$ & $91,89 \pm 17,51$ \\
Altura(mm) & $60,75 \pm 9,87$ & $67,23 \pm 4,29$ & $83,78 \pm 7,70$ \\
Crown Rump $(\mathrm{mm})$ & $117,71 \pm 12,94$ & $141,23 \pm 28,80$ & $161,46 \pm 46,32$ \\
Perímetro ocular $(\mathrm{mm})$ & $9,57 \pm 1,09$ & $10,55 \pm 1,25$ & $11,59 \pm 1,75$ \\
Comprimento auricular $(\mathrm{mm})$ & $14,13 \pm 2,48$ & $17,25 \pm 2,38$ & $20,41 \pm 1,89$ \\
Comprimento abdominal $(\mathrm{mm})$ & $52,70 \pm 4,97$ & $57,49 \pm 8,75$ & $72,54 \pm 6,98$ \\
Diâmetro biparietal $(\mathrm{mm})$ & $30,27 \pm 9,59$ & $33,44 \pm 2,08$ & $38,30 \pm 5,43$ \\
Comprimento torácico $(\mathrm{mm})$ & $43,62 \pm 4,58$ & $51,07 \pm 3,33$ & $62,51 \pm 10,38$ \\
Capacidade volumétrica(mL) & $3,10 \pm 1,48$ & $7,79 \pm 2,61$ & $19,70 \pm 3,78$ \\
\hline
\end{tabular}

Os dados morfométricos neonatais estudados em nossa pesquisa são úteis como parâmetros para identificação de normalidades estruturais e da morfologia externa em neonatos de pequeno, médio e grande porte, além de auxiliar no exame clínico geral feito pelo neonatologias, ainda nas primeiras horas após o parto.

Na prática veterinária, o exame clínico neonatal é uma ferramenta importante na inspeção geral do animal no intuito de se observar alterações fetais (mal formação), lesões durante a parição e/ou a necessidade de manejo diferenciado para evitar a morte ainda na fase de vida inicial. (BARRETO, 2003). Além disso, observou-se que os parâmetros analisados apresentaram uma regularidade para cada porte. Neste sentido, é possível através destes dados uma classificação dos neonatos em três portes, não apenas segundo o peso, mas também segundo a morfometria.

A sintopia do estômago no neonato está representada na figura 2 onde observa-se que, apesar do pequeno tamanho em relação aos adultos, foi possível observar todas as estruturas que compõem o estômago do canino adulto. (GETTY,1986). Desta forma, o cárdia, na transição do esôfago e estômago, apresentou-se como uma banda circular e de curta extensão. $O$ fundo volumoso e arredondado projeta-se dorsalmente à esquerda da cárdia contra o fígado, tornandose a parte mais dorsal do órgão. Este situa-se principalmente à esquerda do plano mediano em contato com o diafragma e o fígado. O fígado ocupa grande parte da cavidade abdominal do neonato. Á medida que se aproxima da parte pilórica 0 fundo afunila-se para formar o corpo que desemboca na parte pilórica. A extremidade pilórica é pequena e está direcionada cranial e dorsalmente; ela 
normalmente está opostamente situada à parte ventral da nona costela ou espaço intercostal e a uma distância variável para a direita do plano mediano. Ela está relacionada com a fissura portal do fígado e com o pâncreas.

Nos neonatos estudados, observamos que todas as estruturas de ligação estão presentes, sendo que o omento maior apresentou-se bastante resistente. Esta característica possibilitaria uma conexão mais estreita com o baço, órgão ligado a imunologia nos cães. O neonato, por possuir características imunológicas ainda em desenvolvimento, necessita de estratégias que facilitem sua sobrevivência e esta possivelmente é uma delas. Segundo DYCE et al. (1997), o fundo é diretamente ligado ao pilar esquerdo do diafragma (ligamento gastrofrênico), enquanto existem ligações mais frouxas entre o cárdia e o diafragma, a curvatura menor do fígado (omento menor) e curvatura maior do baço (omento maior). Exceto nessas reflexões, o estômago é totalmente revestido por serosa.

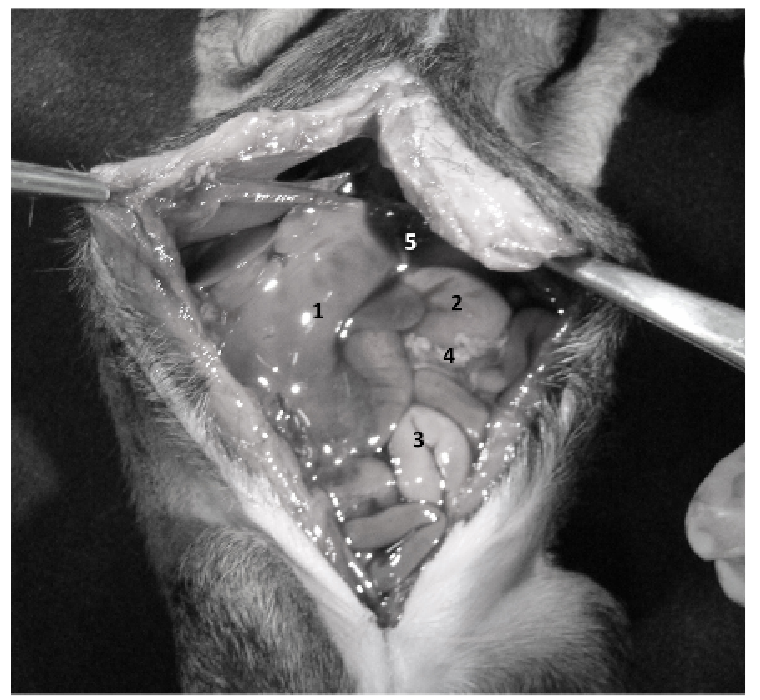

FIGURA 2: Sintopia do estômago na cavidade abdominal de neonato canino de médio porte. 1-Fígado; 2-estômago; 3Intestino delgado; 4-Omento maior; 5Diafragma. Fonte: Arquivo Pessoal.

Para GETTY (1986), a cárdia apresenta-se como uma abertura maior que o abertura do piloro e esta amplificação pode estar relacionada com a facilidade com que os cães vomitam. Nos cães neonatos estudados, esta amplificação também é notável, o que sugere que neste período etário também é possível regurgitações. Neste caso, não só a facilitação pela abertura extensa da cárdia, como também uma alimentação em excesso, é fator importante para que ocorra este fenômeno.

A parte pilórica do neonatos estudados é também afetada pelas variações na quantidade ingerida e atinge o assoalho abdominal apenas quando o estômago está muito dilatado (GETTY, 1986). Em nosso estudo percebeu-se que esta porção do estômago apresentou-se bastante muscular.

Quanto a capacidade volumétrica, observou-se que em neonatos caninos existe um aumento gradativo na capacidade volumétrica dependente do porte do animal (Figura 3). Sendo que, os neonatos caninos de grande porte apresentaram a média volumétrica mais regular. 


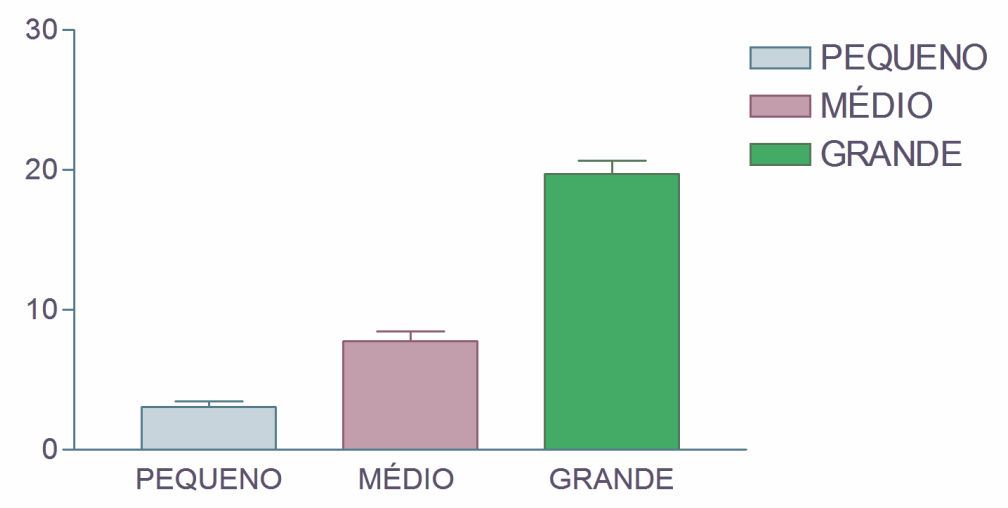

FIGURA 3: Gráfico representando as médias das capacidades volumétricas entre pequeno, médio e grande porte de neonatos caninos.

De acordo com o peso, estudos indicam a capacidade como sendo de 100 a $250 \mathrm{~mL} / \mathrm{Kg}$ de peso do animal, ou ainda, estimam a capacidade em cerca de 1 litro para um canino de peso médio (aproximadamente 10Kg). (GETTY, 1986). Essa relação entre peso e capacidade volumétrica feita para o canino adulto não é a mesma em neonatos, conforme nossos dados encontrados. Se usássemos a mesma relação, em cães neonatos de médio porte (com cerca de $300 \mathrm{~g}$ ) a capacidade volumétrica nestes animais seria de $30 \mathrm{~mL}$, quando, o identificado em nossos estudos, para neonatos de médio porte (com cerca de $232 \mathrm{~g}$ ) foi de $7,79 \mathrm{~mL}$, em média, com desvio de $\pm 2,61 \mathrm{~mL}$. Isso demonstra que existe uma ineficiência em relação à relação peso e capacidade volumétrica, já que não existem estudos relacionados para cães neonatos.

Um filhote canino deve duplicar seu peso em 10 a 12 dias de vida, com um aumento diário em média de $2 \mathrm{~g}$ para cada quilo de peso calculado para um adulto. (DOMINGOS et al., 2008). Sendo assim, um neonato de porte médio encontrado em nossos estudos, aumentaria cerca de vinte gramas por dia e seu peso duplicaria em cerca de 11 dias, o que confirma os dados da literatura.

Assim, dependendo do porte do animal, sua capacidade volumétrica aumentaria e ainda, com o passar dos dias sua dieta teria de ser adaptada a um aumento de peso do animal. A falha no manejo da quantidade de alimento adequado está entre uma das principais causas de morte para animais neste período de vida. (BRUNETTO, 2006). Por esse motivo, é necessário um acompanhamento adequado às necessidades do animal.

\section{CONCLUSÕES}

A sintopia do estômago de neonatos caninos e a morfologia externa não difere dos demais animais adultos. Os dados morfométricos encontrados servem como parâmetros para normalidades, auxiliando os neonatologistas veterinários, nos primeiros cuidados neonatais. Além disso, foram observadas diferenças entre o volume de alimento que deve ser administrado no período neonatal para os gêneros e os portes variados, sendo neste último, diretamente proporcional. Este estudo auxilia na elucidação da capacidade volumétrica adequada e, neste sentido, o 
médico veterinário deve atentar-se para o tempo conveniente entre a administração de alimento para os neonatos caninos.

\section{REFERÊNCIAS}

AIRES, M.M. Fisiologia. 3.ed. Rio de Janeiro:Guanabara Koogan, 2008.

BARRETO, C.S. Avaliação de filhotes caninos. 2003. 19f. Monografia(Doutorado) - Faculdade de Medicina Veterinária e Zootecnia - Universidade de São Paulo, 2003.

BERCHT, B.S. Úlcera de córnea profunda em cães. 2009. $35 f$. Monografia(Graduação) - Faculdade de Veterinária - Universidade federal do Rio Grande do Sul, 2009.

BURBIDGE, H.M; THOMPSON, K.C.; HODGE, H. Post natal development of canine caudal cervical vertebrae. Vet Sci. v.59, n.1, p.35-40, 1995.

BRUNETTO, M.A. Avaliação de suporte nutricional sobre a alta hospitalar em cães e gatos. 2006. 103f. Dissertação(Mestrado) - Faculdade de Ciências Agrárias e Veterinárias - Universidade Estadual Paulista, 2006.

CARVALHO, Y.S.V.; XEREZ, D.R.; ARAÚJO, A.Q.C. Identificação de broncoaspiração por disfagia orofaríngea em pacientes com pneumonia comunitária. Acta Fisiatr v.13, n.2, p.59-62, 2006.

CHAVES Jr., N.; MAGALHÃES, L. T.; COLLEONI, R.; Del GRANDE, J. C. Efeitos da elevação da pressão intra-abdominal e de seu tempo de ação na cicatrização de suturas mecânicas no estômago de cães. Act Cirug Bras. v. 22, n.5, p.379-386, 2007.

CRESPILLO, A. M.; MARTINS, M. I. M; SOUSA, F. F; LOPES, M. D; PAPA, F. O. Abordagem terapeutica do paciente neonato canino e felino: 2. Aspectos relacionados a terapia intensiva, antiparasitarios e antibioticos. Rev Bras Reprod Anim, Belo Horizonte, v.31, n.4, p.425-432, 2007.

DAVIDSON, A.P. Approches to reducing neonatal mortality in dogs: In: CONCANNON PW, ENGLAND G., VERSTEGEN III.J, LINDE-FORSBERG. Recent Advances in Small Animal Reproduction. New York, 2003.Disponível em:< http://www.ivis.org> Acesso em 27 de outubro de 2010.

DYCE, K. M; SACK, W. O; WENSING, C. J. G. Tratado de Anatomia Veterinária. 2 ed. Rio de Janeiro: Guanabara Koogan, 1997.

DOMINGOS, T.C.S.; ROCHA, A.A; CUNHA, I.C.N. Cuidados básicos com a gestante e o neonato canino e felino: Revisão de literatura. J. Bras de Cien Anim. v.1, n.2, p. 94-120, 2008.

FURNISS, G. Obesidade canina: como mudanças de comportamento podem ajudar a evitá-la. Focus Aux. v.1, n. 2, p.1-32, 2010. 
GETTY, R. Sisson e Grossman Anatomia de animais domésticos. 5. ed. vol. 2. Rio de janeiro: Guanabara koogan, 1986.

GUYTON, A.C.; HALL, J.E. Tratado de Fisiologia Médica. 10 ed. vol.1. Rio de Janeiro: Guanabara Koogan, 2002.

INDREBO, A; TRANGERUD, C; MOE, L. Canine neonatal mortality in four large breeds. Act Vet Scand. v.49 (Suppl 1):S2, 2007.

KERSWELL, K.J.; BENNETT, P.; BUTLER, K.L.; HEMSWORTH, P.H. The relationship of adult morphology and early social signaling of the domestic dog (Canis familiaris). Behav. Proc. v.81, p.376-382, 2009.

MANDARIM - DE - LACERDA, C.A. Manual de qualificação morfológica: morfometria, alometria, estereologia. Rio de Janeiro, p. 05 - 16. 1994.

OLIVEIRA, A.G.; SIQUEIRA, P.P.; ABREU, L.C.; Cuidados Nutricionais no Recémnascido de Muito Baixo Peso. Rev Bras Crescimento Desenvol Hum., v.18, n.2, p.148-154, 2008.

PASSOS, J. F; Trabalho de conclusão do curso de medicina veterinária. 2006. $55 f$ (Monografia)- Aréa de obstetrícia veterinária. Faculdade Integradas, Brasília, 2006.

TORTOLA, L. Alimentação e nutrição de cães e gatos neonatos. Anim de Comp. v.1, n.1, p.6-7. 2008. 